\title{
Crystals 2020
}

\section{2 crystals TMPI}

\section{Optics of cholesterics with oblique helicoidal director}

Olena S. Iadlovska ${ }^{12 *}$, Mateusz Mrukiewicz ${ }^{13}$, Kamal Thapa ${ }^{12}$, Greta Babakhanova ${ }^{1}$, Graham R. Maxwell ${ }^{1}$, Sergij V. Shiyanovskii ${ }^{1}$, and Oleg D. Lavrentovich ${ }^{12}$

${ }^{1}$ Advanced Materials and Liquid Crystal Institute, and

${ }^{2}$ Department of Physics, Kent State University, Kent, OH 44242, USA

${ }^{3}$ Institute of Applied Physics, Military University of Technology, 00-908 Warsaw, Poland

* Corresponding author: oiadlovs@ kent.edu
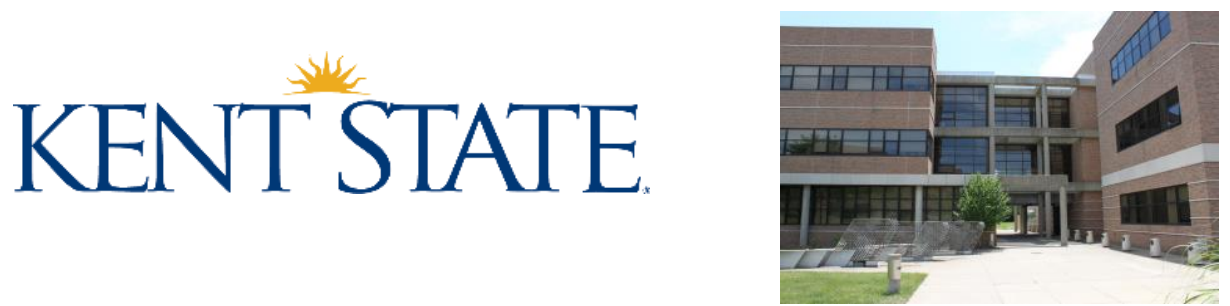


\section{Outline}

- Oblique helicoidal cholesteric $\left(\mathrm{Ch}_{\mathrm{OH}}\right)$ state as continuously tunable 1D photonic crystal

- Effect of the surface alignment on Bragg reflection

- In-situ measurement of bend elastic constant in Ch phase

- Bragg diffraction at oblique incidence; polarization dependency 


\section{Cholesterics as 1D photonic crystals}

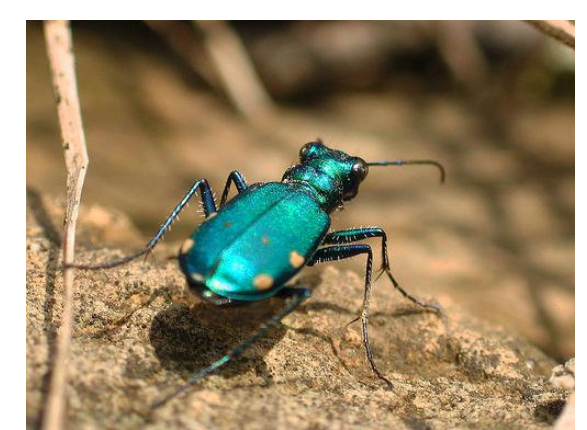

Naturally occurring colors - result of Bragg reflection at the periodic structure with the period close to the wavelength of visible light.

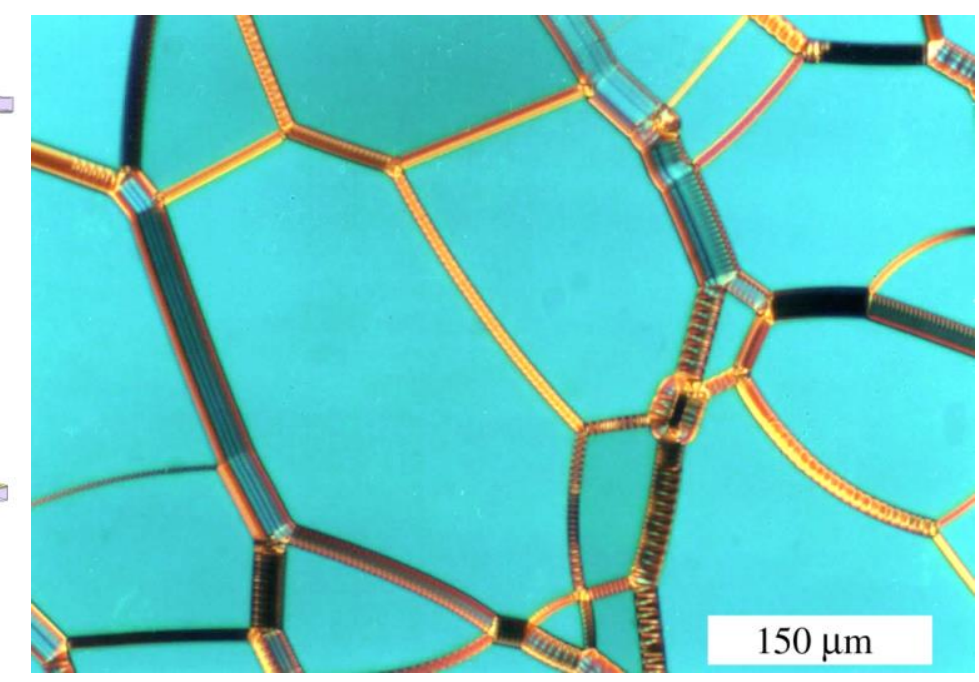

Polarizing microscope texture of the cholesteric liquid crystal $\lambda_{0}-$ Bragg wavelength

$\Delta \lambda-$ Bandwidth, or width at the half-amplitude

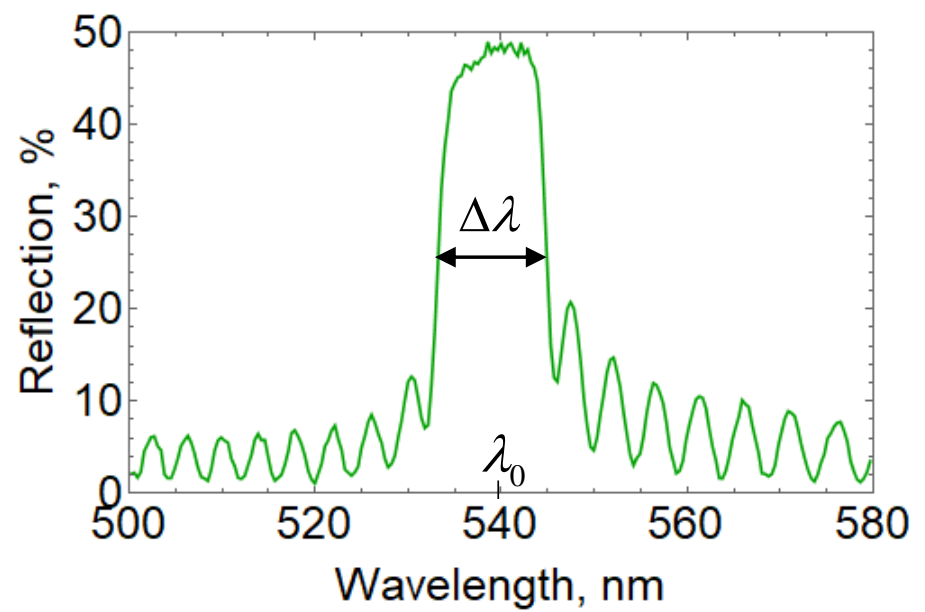

$$
\begin{gathered}
\Delta \lambda=\Delta n \frac{P_{0}}{2} \\
\lambda_{0}=\bar{n} \frac{P_{0}}{2}
\end{gathered}
$$




\section{Cholesterics with positive dielectric anisotropy, $\Delta \varepsilon>0$}

Attempt to control the pitch by the applied field $\mathbf{E}$ leads to distortion of the helical structure and transformation to a nematic state $(\mathrm{N})$ with the director $\hat{\mathbf{n}}$ along the field (here, $\hat{\mathbf{h}}$ is the helix axis).

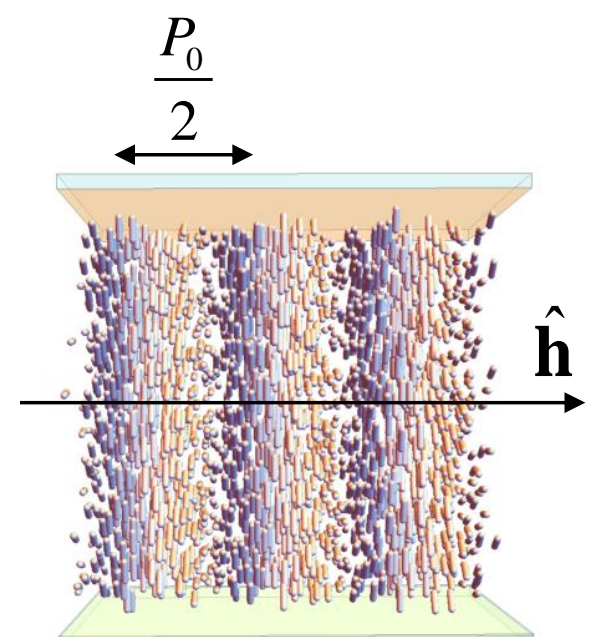

Ch cell

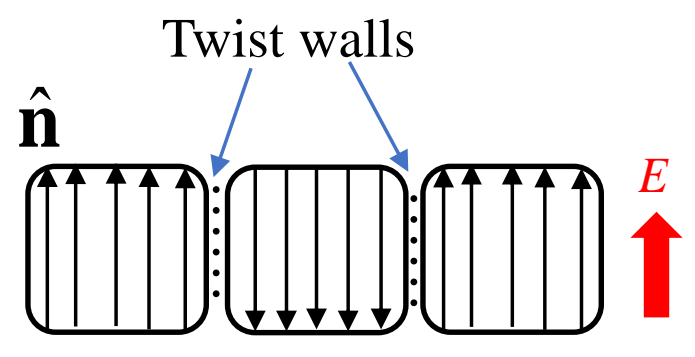

Single-harmonic modulation of the director is destroyed under the action of applied field

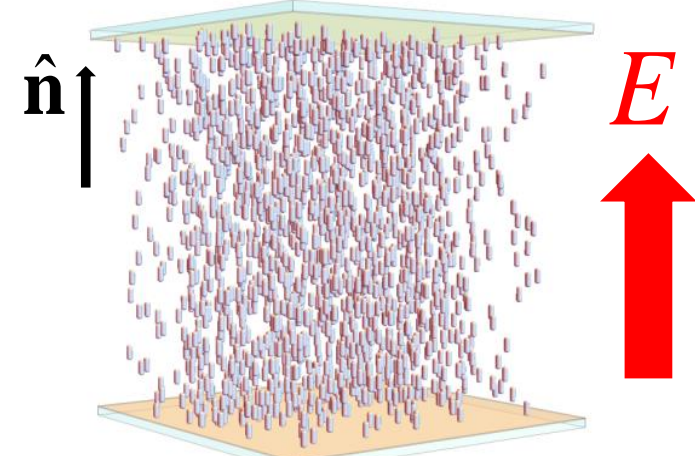

Transition to a nematic state

Material: rod like molecules which are hard to bend, i.e. bend elastic constant $K_{33}$ is large as compared to twist $K_{22}$ and splay $K_{11}$ moduli. Ch period is continuously tuned by the temperature, but not by applied field. Common example: 5CB molecule

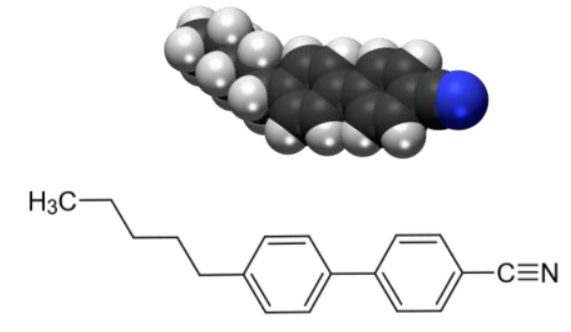

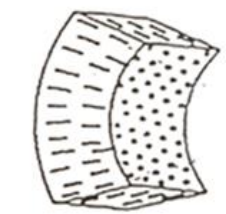

Splay
$\mathrm{K}_{11}$

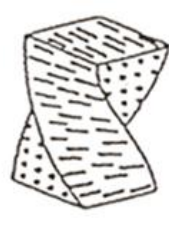

Twist

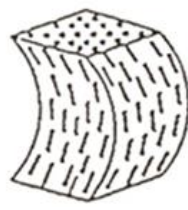

Bend

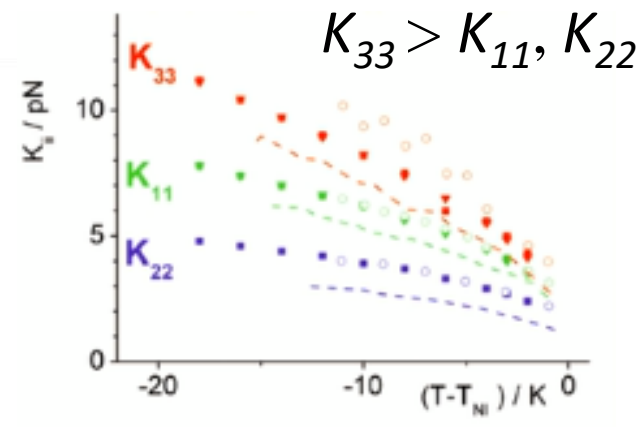

Ferrarini et al, J. Chem. Phys. 131, 054104 (2009) 


\section{Oblique helicoidal cholesteric $\left(\mathrm{Ch}_{\mathrm{OH}}\right)$ state, $\Delta \varepsilon>0$}

Theoretical prediction:

R. B. Meyer, Applied Physics Letters 12, 281 (1968). ;

P. G. De Gennes, Solid State Communications 6, 163 (1968).

\section{Experimental observation:}

PRL 112, 217801 (2014)

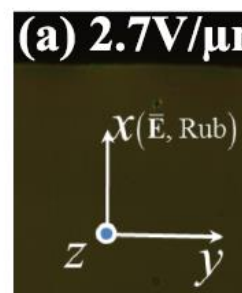

\section{$50 \mu \mathrm{m}$}

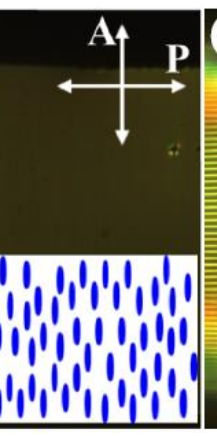

\section{(b) $0.5 \mathrm{~V} / \mathrm{\mu m}$}
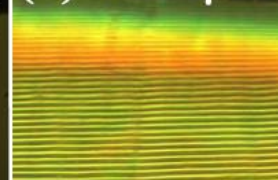

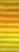

\section{0, $\quad 1,1,1,1,1,1$}

$E_{C h}<E_{C h O H}<E_{N}$ when $K_{33} / K_{22} \leq 0.5$

$\mathrm{Ch}_{\mathrm{OH}}$ pitch $P$ and cone angle $\theta$ are both continuously tunable by the applied field $E_{\mathrm{ChOH}}$

$K_{22}$ and $K_{33}$ are elastic constants of twist and bend

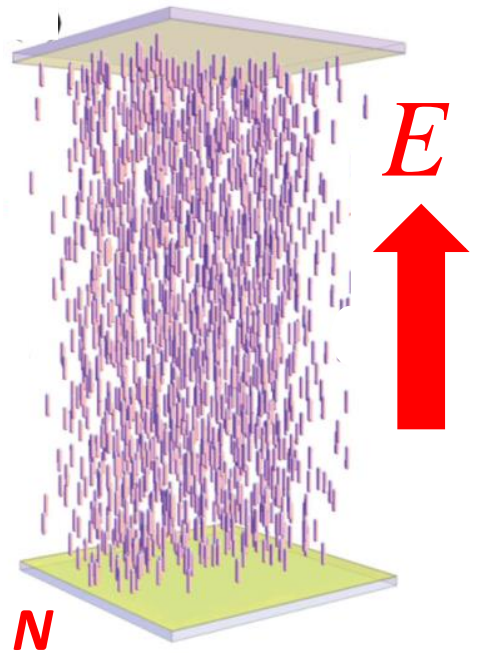

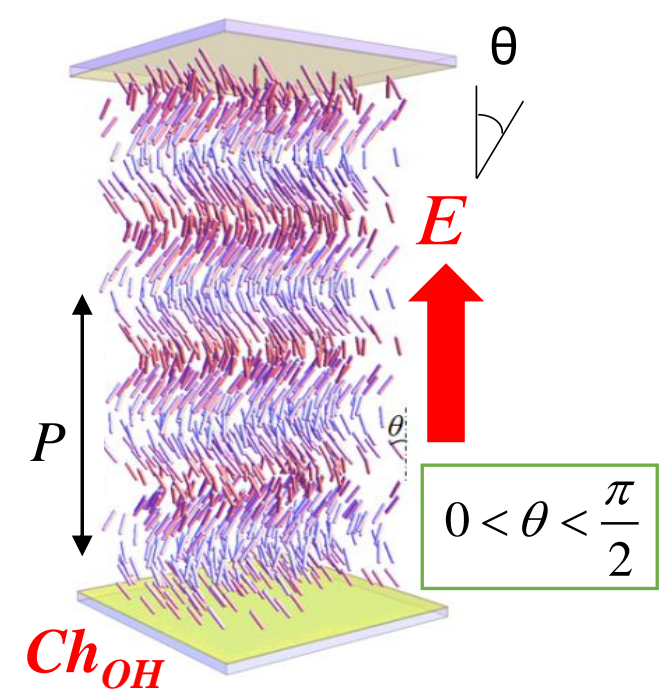

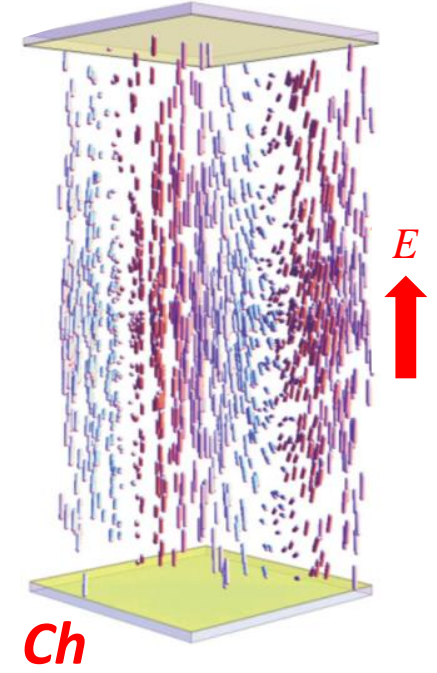

\section{(c) $0.3 \mathrm{~V} / \mu \mathrm{m}$}

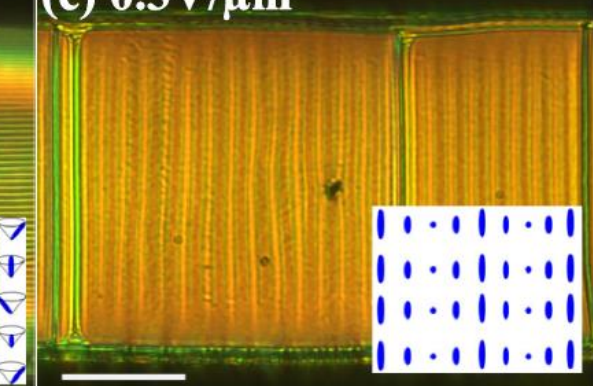

J. Xiang et al, Phys Rev Lett 112(21), 217801 (2014).
Material:

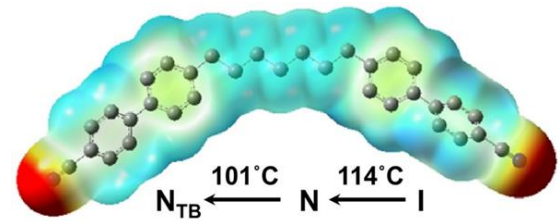

Flexible dimer $1^{\prime \prime}, 7^{\prime \prime}$ - bis(4-cyanobiphenyl-4'-yl) heptane $(\mathrm{CB} 7 \mathrm{CB})$

is easy to bend (small $\kappa_{33}$ )

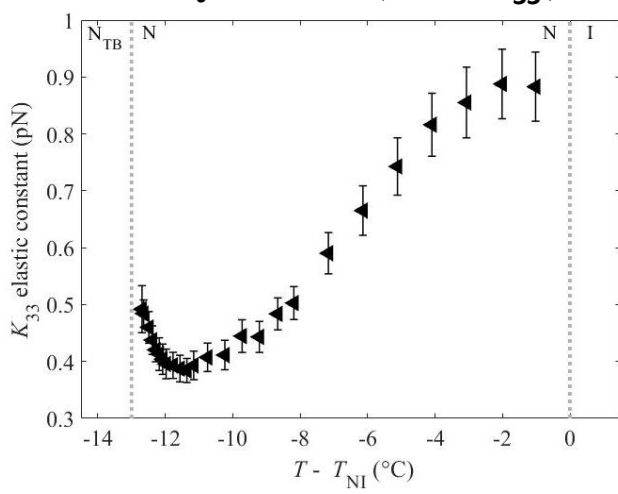

G. Babakhanova, Z. Parsouzi et al, Phys Rev E 96, 062704 (2017) 


\section{$E$-tuned Bragg reflection at normal incidence of light}

$$
\begin{aligned}
& \text { IIt. } \\
& \lambda_{\text {Bragg }}=\bar{n} P, \quad \bar{n}=\frac{\left(n_{e}^{\text {eff }}+n_{o}\right)}{2} \\
& n_{e}^{e f f}=\frac{n_{e} n_{o}}{\sqrt{n_{e}^{2} \cos ^{2} \theta+n_{o}^{2} \sin ^{2} \theta}}
\end{aligned}
$$

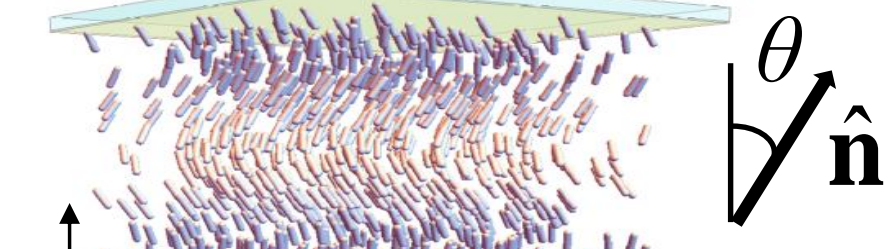

$$
\Delta \lambda=\frac{K_{33}^{2} E_{N} P_{0} n_{o}}{2 E K_{22}\left(K_{22}-K_{33}\right)}\left(1-\frac{n_{o}^{2}}{n_{e}^{2}}\right)\left(\frac{E_{N}}{E}-1\right)
$$

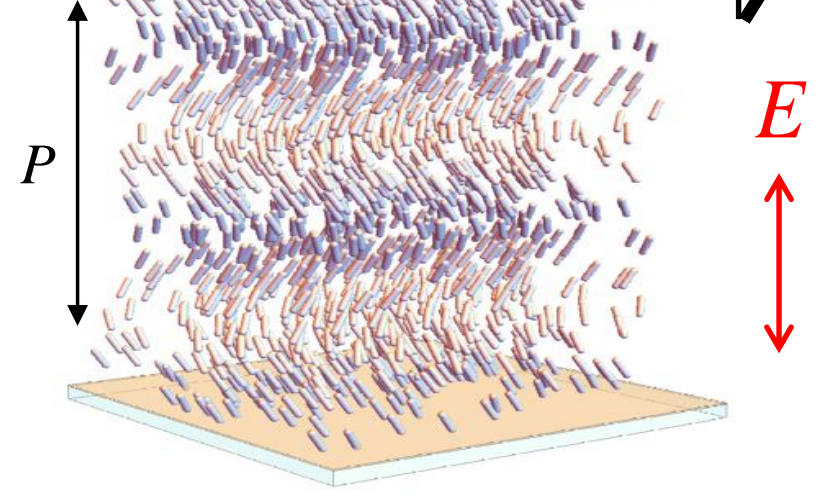

$$
P=\frac{2 \pi}{E} \sqrt{\frac{K_{33}}{\varepsilon_{0} \Delta \varepsilon}}
$$

$$
\sin ^{2} \theta=\frac{\kappa}{1-\kappa}\left(\frac{E_{N}}{E}-1\right) ; \quad \kappa=\frac{K_{33}}{K_{22}}
$$

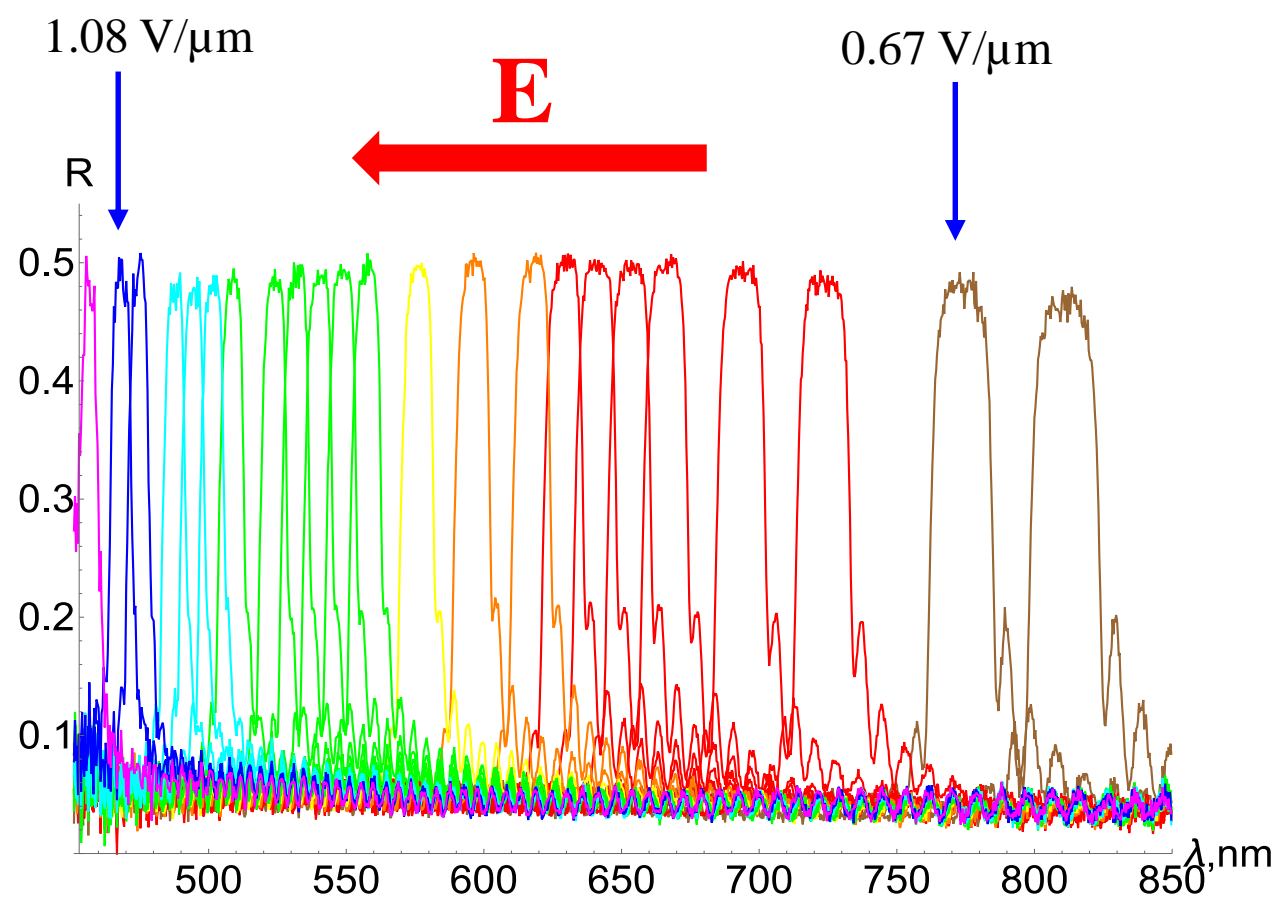




\section{At constant applied field, reflection wavelength is different in planar and homeotropic cells}
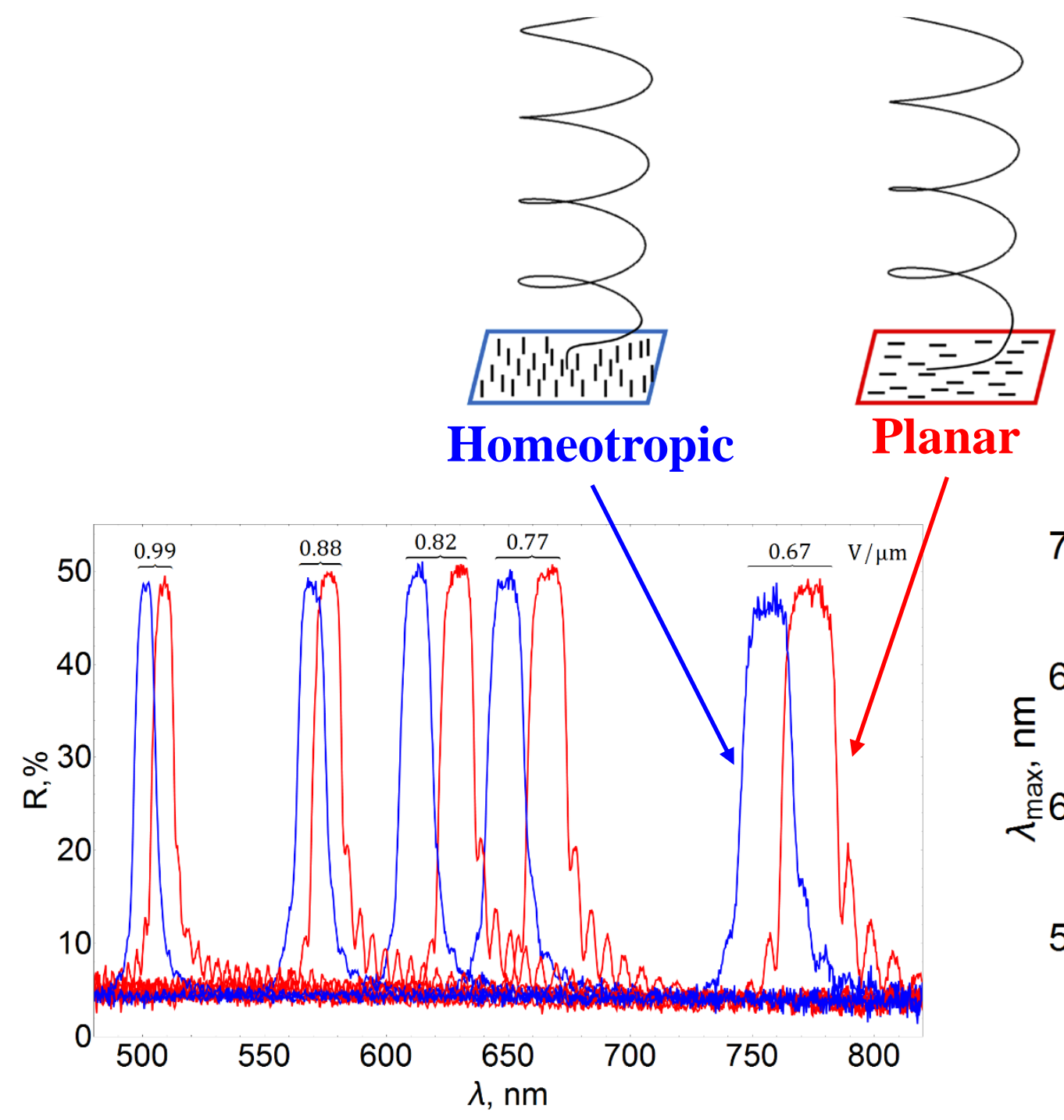

At the same value of the field, spectra in homeotropic $\mathrm{Ch}_{\mathrm{OH}}$ cells are blue-shifted, and in the planar $\mathrm{Ch}_{\mathrm{OH}}$ cells are red shifted.

Can be used in sensing applications

Homeotropic 


\section{Dielectric properties are not uniform near substrates}

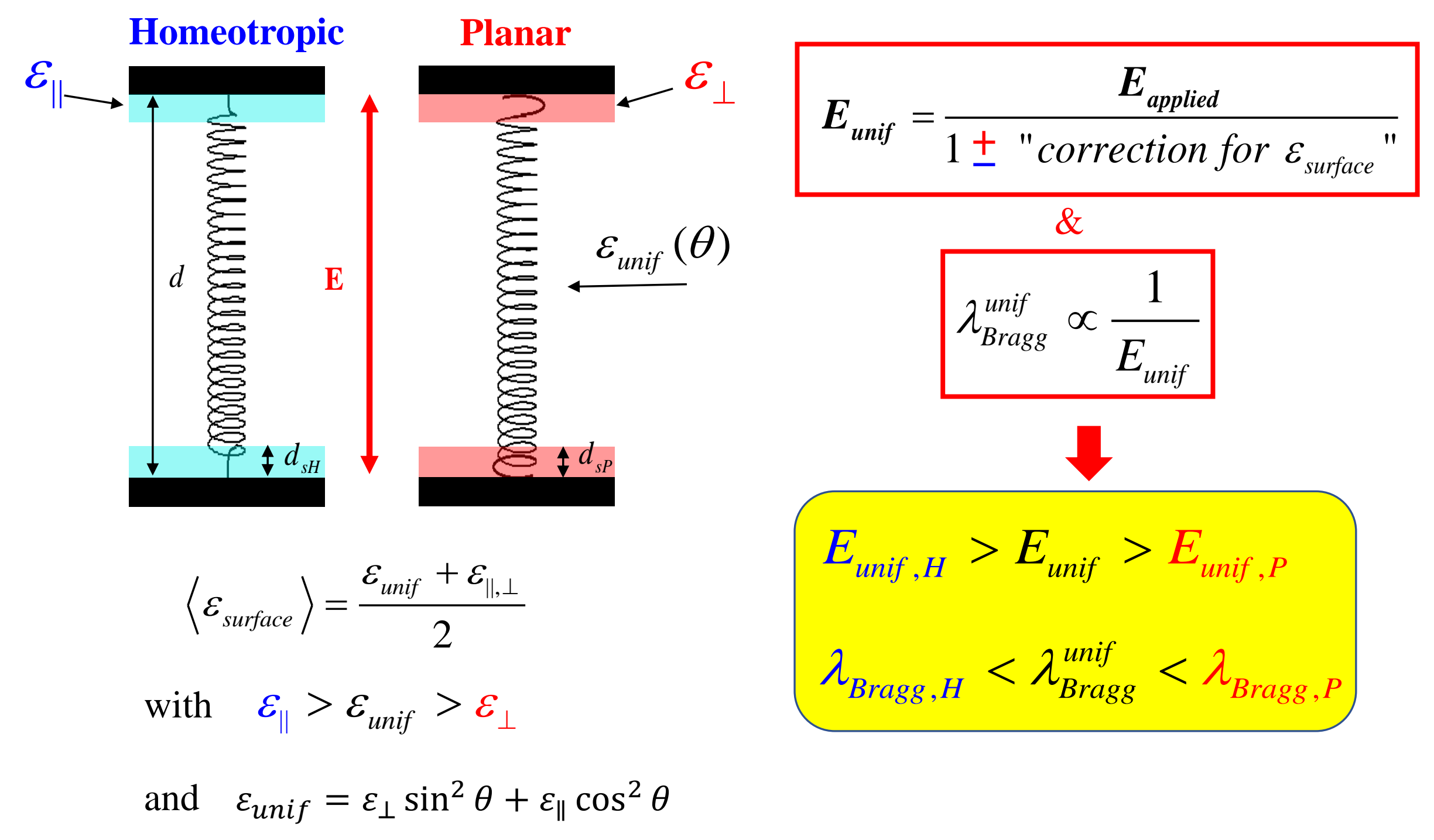




\section{Peaks separation is growing towards the red part of spectrum}

\section{Experiment}
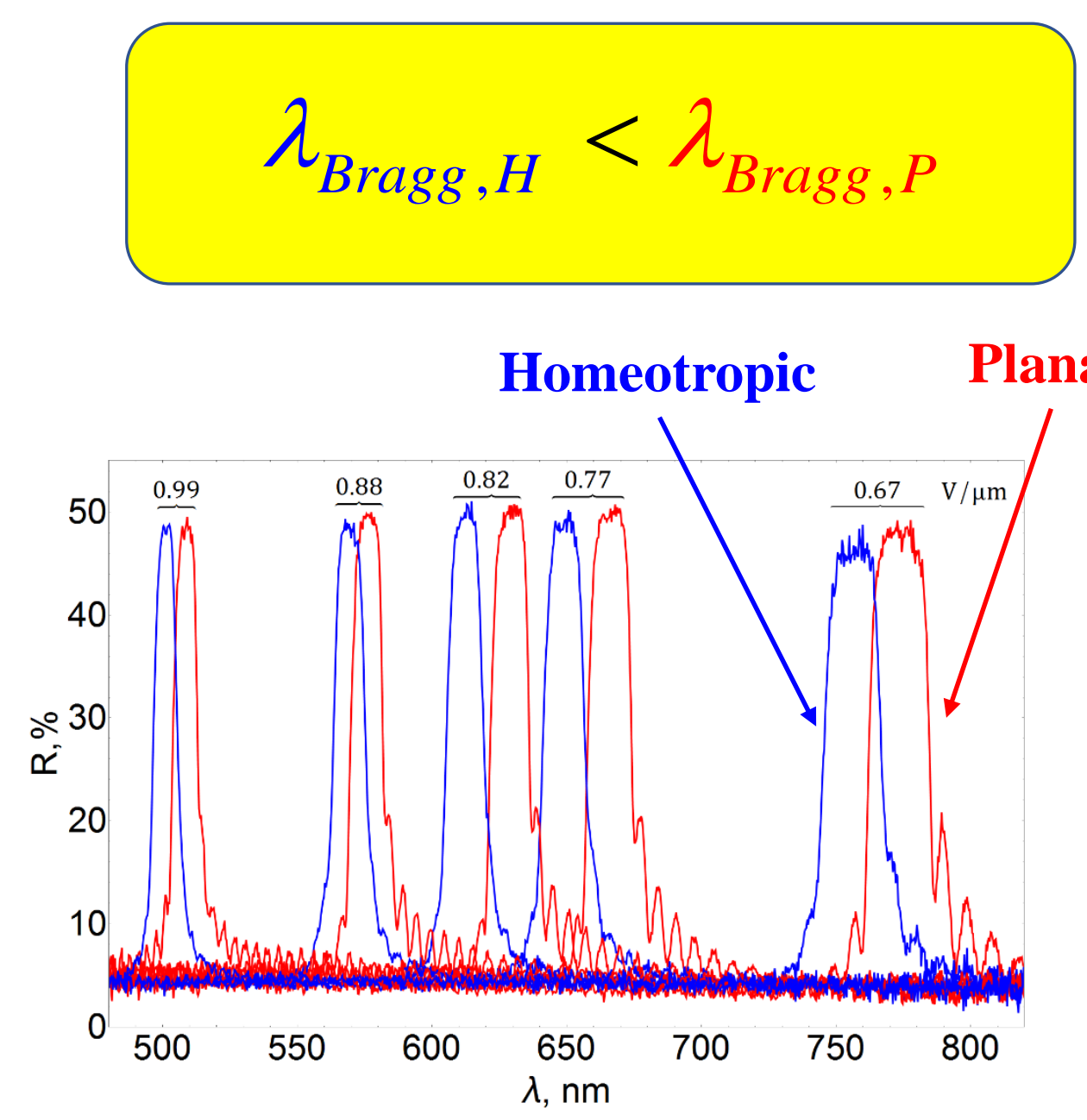

Iadlovska, O.S., et al., Optics Letters, 43(8), 1850 (2018).

\section{Simulations}

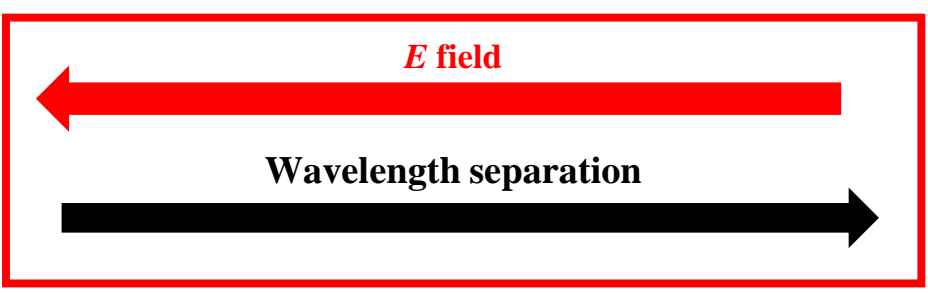

$\mathrm{Ch}_{\mathrm{OH}}$ director model quantifies the director field difference in the two cells

\section{Homeotropic Planar}

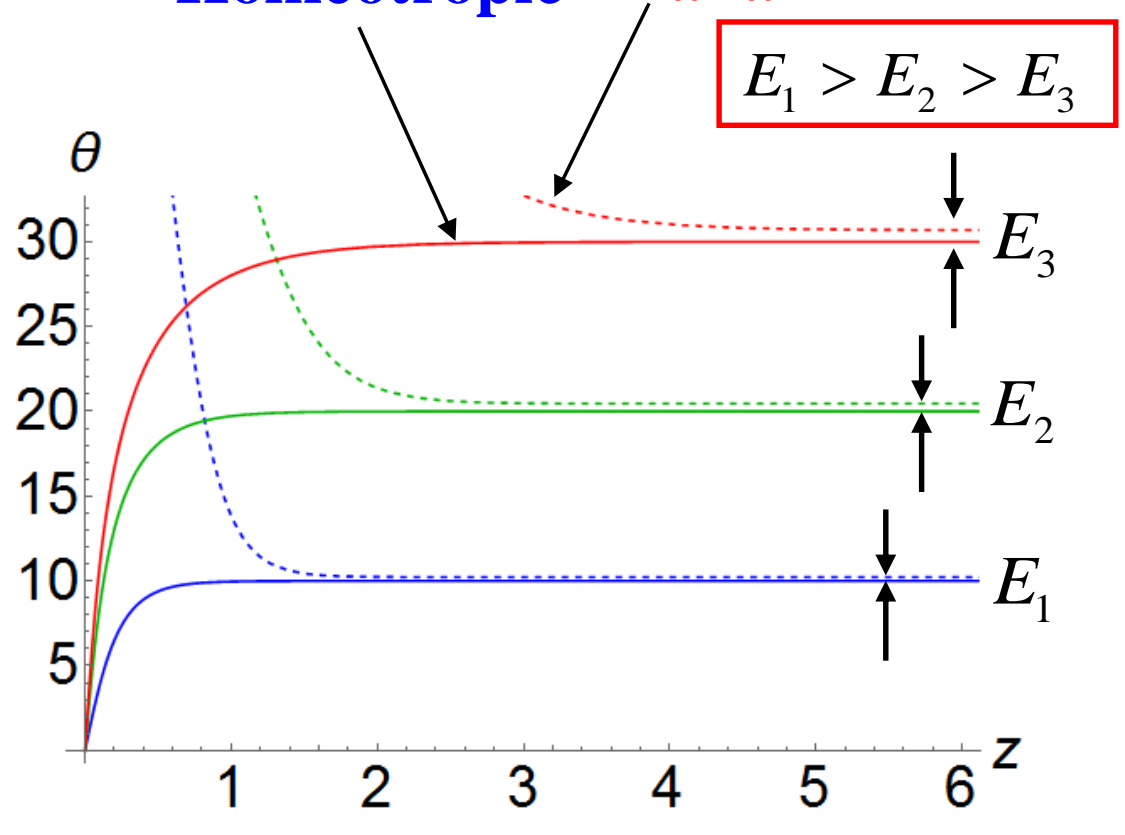




\section{Elasticity of the flexible dimer CB7CB, the first $\mathbf{N}_{t b}$ material}

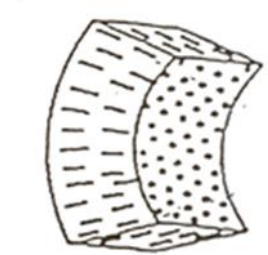

Splay

$\mathrm{K}_{11}$

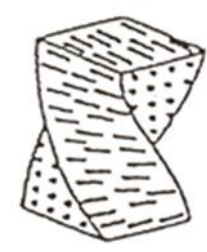

Twist

$\mathrm{K}_{22}$

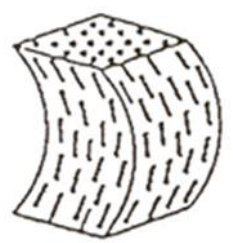

Bend

$\mathrm{K}_{33}$

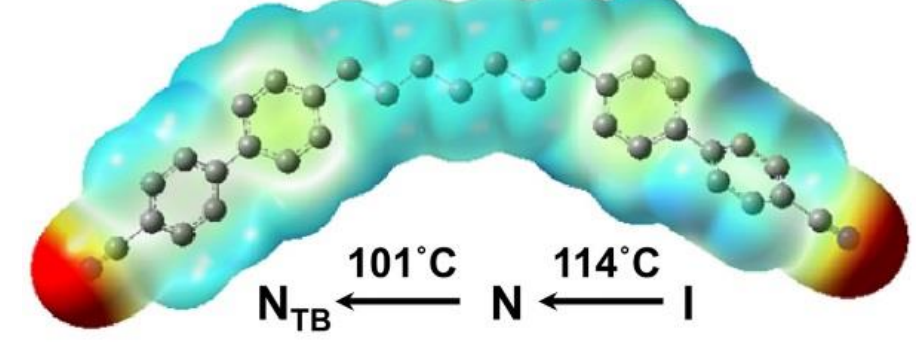

Flexible dimer CB7CB is easy to bend ( (small $K_{33}$ )

Elastic constants of Splay $K_{11}$, Twist $K_{22}$, and Bend $K_{33}$ measured in nematic phase:
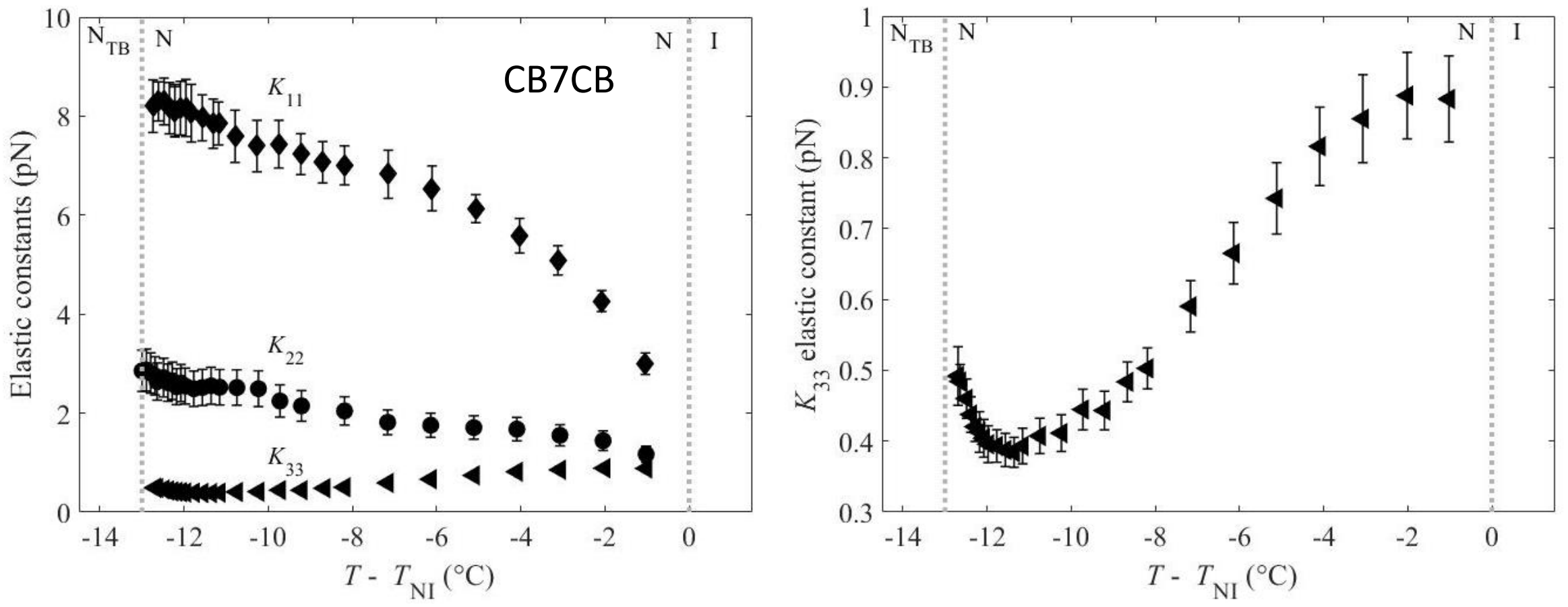

G. Babakhanova, Z. Parsouzi et al, Phys Rev E 96, 062704 (2017) 


\section{In-situ measurement of bend elastic constant in $\mathrm{Ch}$}

Values of $K_{33}$ are deduced from the optical spectra.
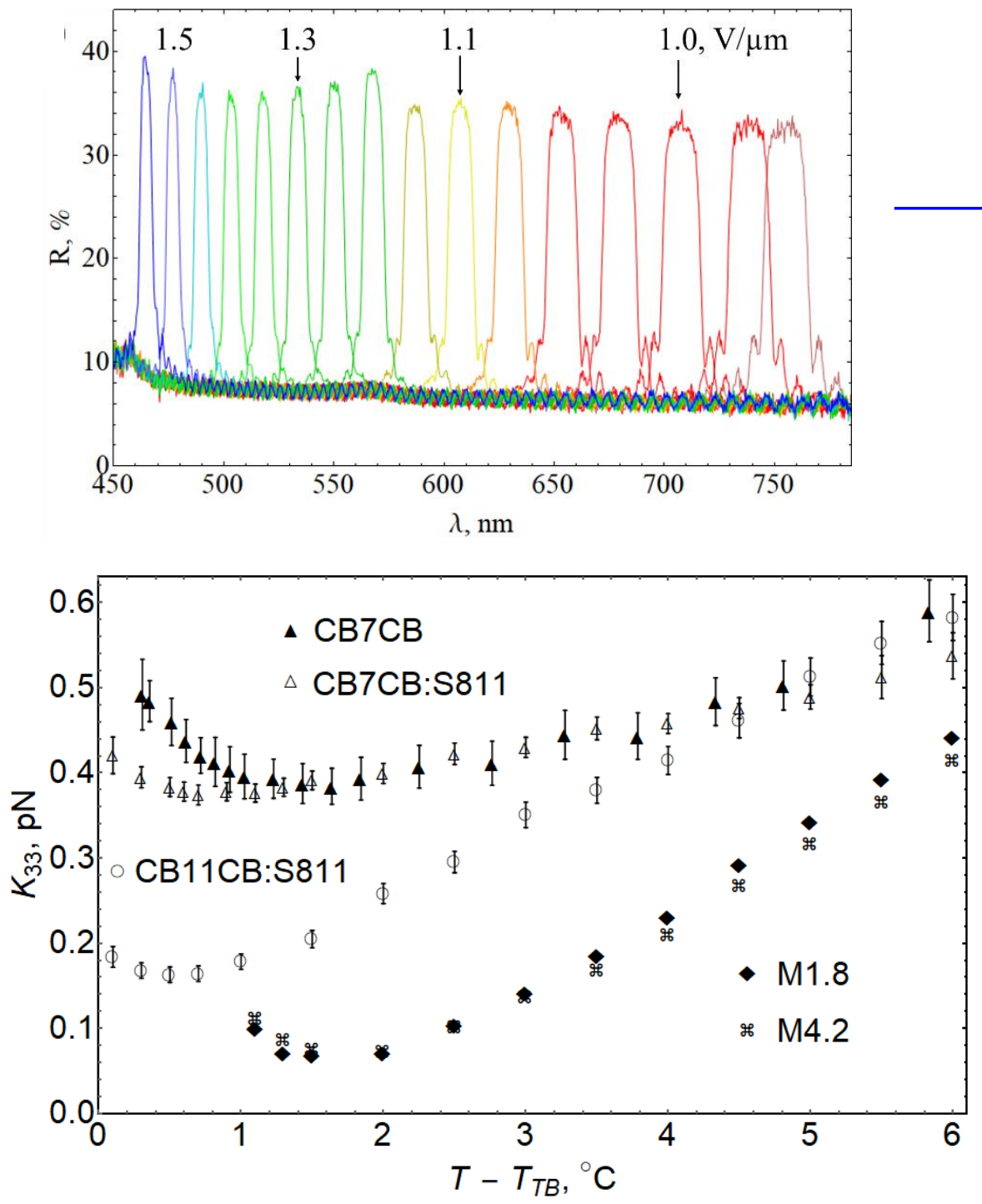

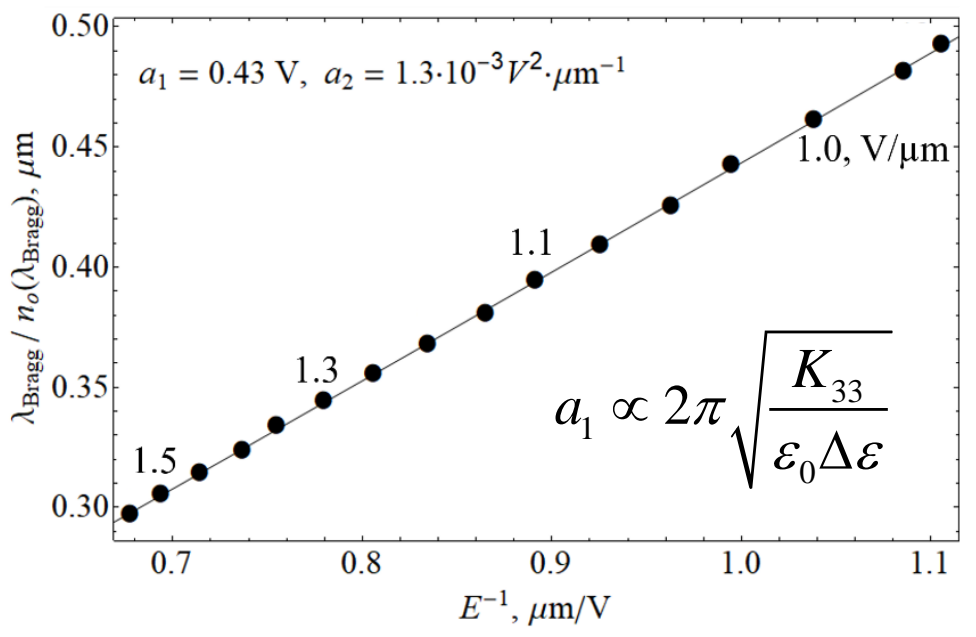

$$
\frac{\lambda_{\text {Bragg }}}{n_{o}}=\frac{2 \pi}{E} \sqrt{\frac{K_{33}}{\varepsilon_{0} \Delta \varepsilon}}
$$

Longer flexible dimer CB11CB has smaller bend modulus as compared to shorter $\mathrm{CB} 7 \mathrm{CB}$ molecule.

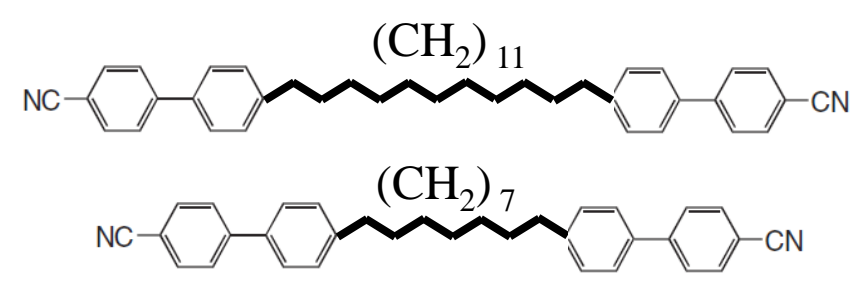




\section{Oblique incidence at $\mathrm{Ch}_{\mathrm{OH}}$ structure}

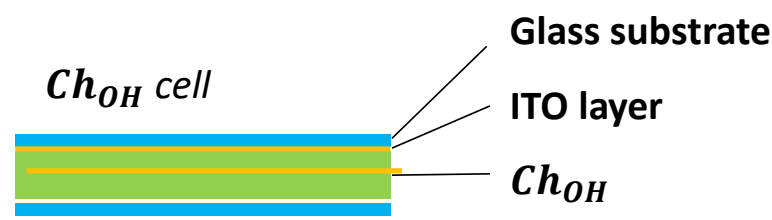

Oblique incidence setup in transmission mode

Light source

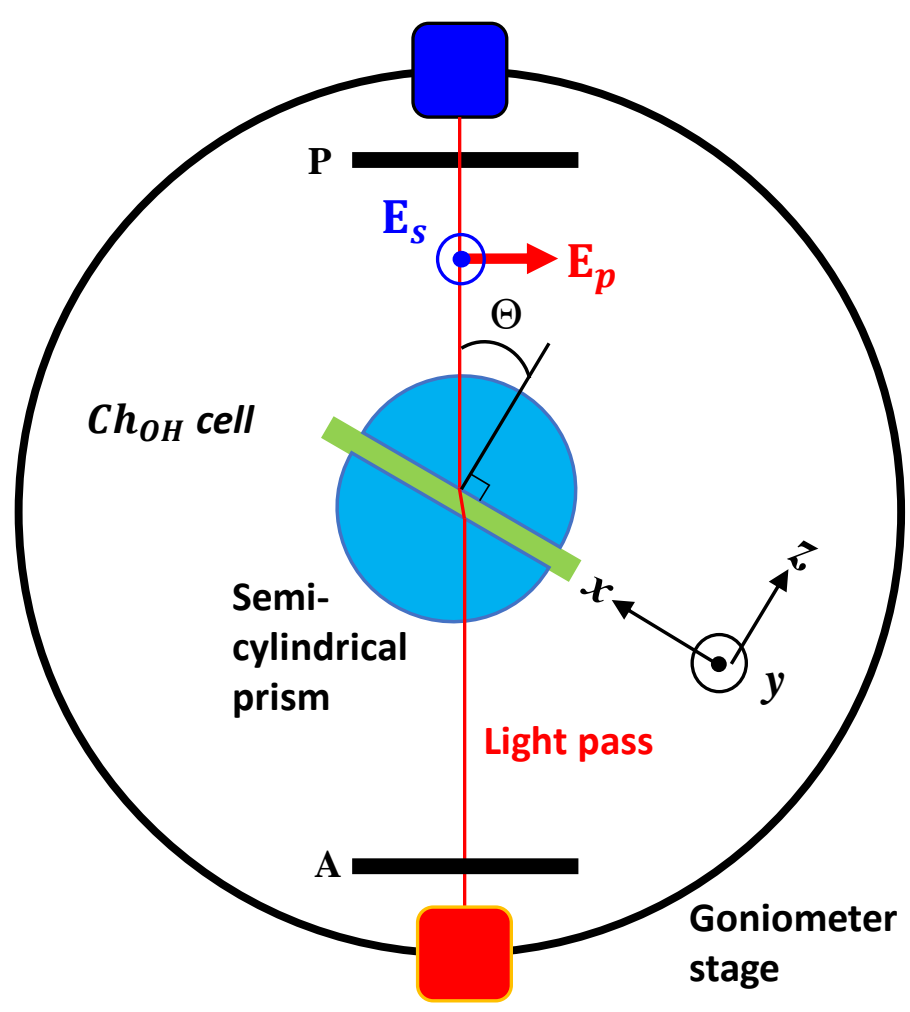

Detector
Diffraction from $\mathrm{Ch}_{\mathrm{OH}}$ structure

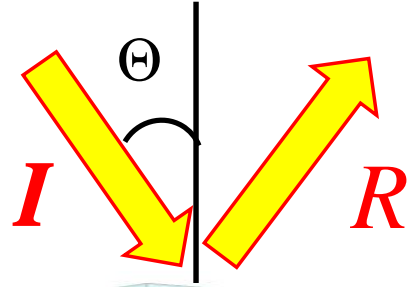
at oblique incidence of light reveals forbidden bands associated with the full pitch $P$ and the half-pitch $P / 2$
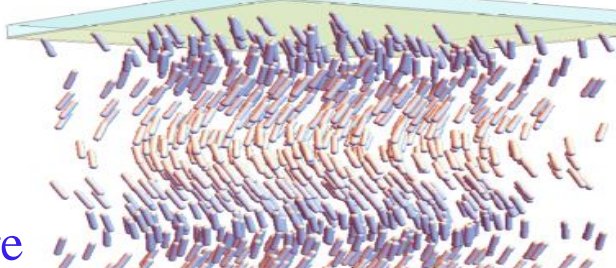

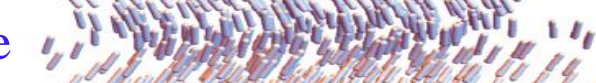
$\gamma^{\theta} \hat{\mathbf{n}}$ E
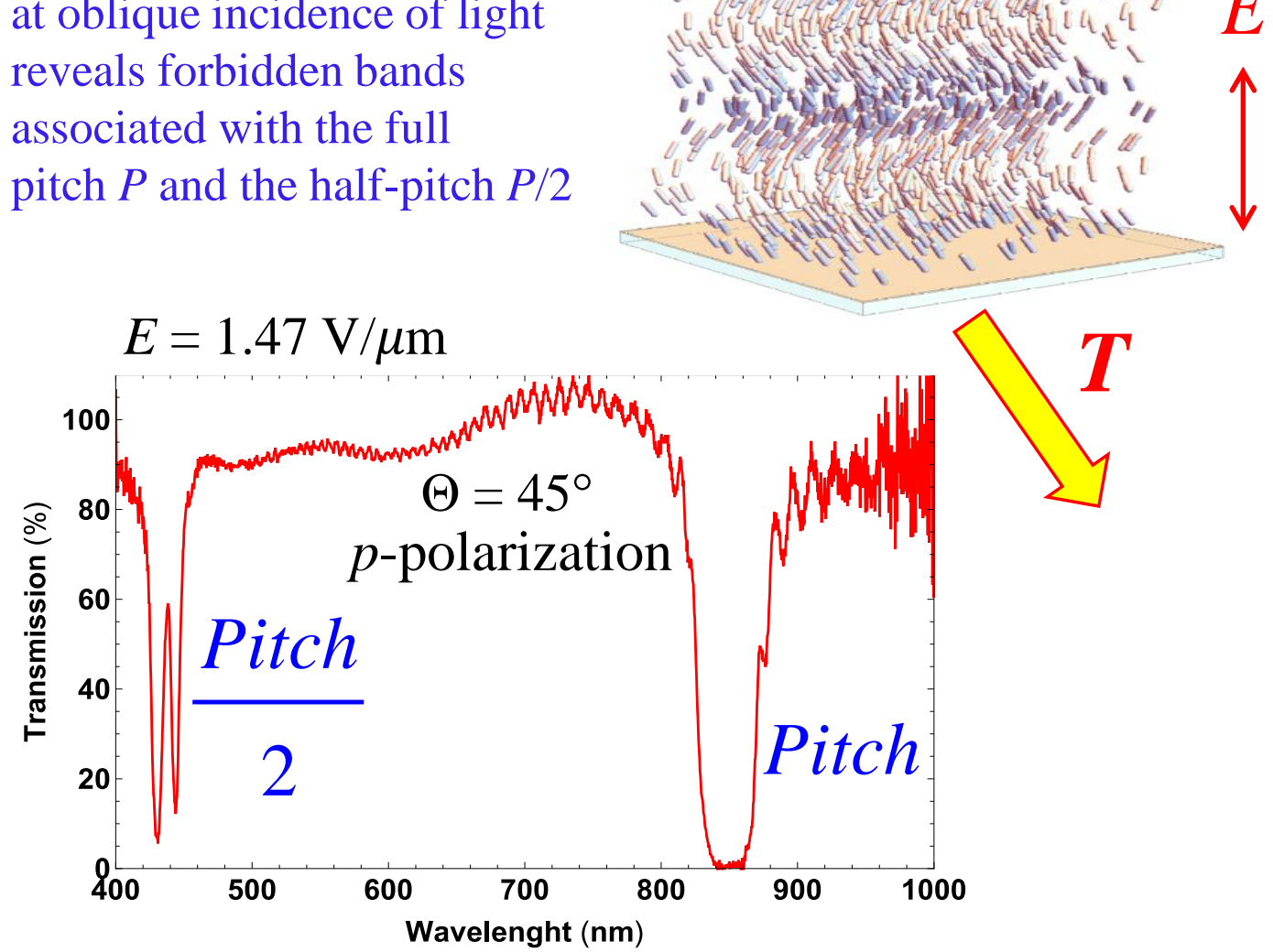


\section{$E$ - and $\Theta$-tuned Bragg diffraction at oblique incidence}
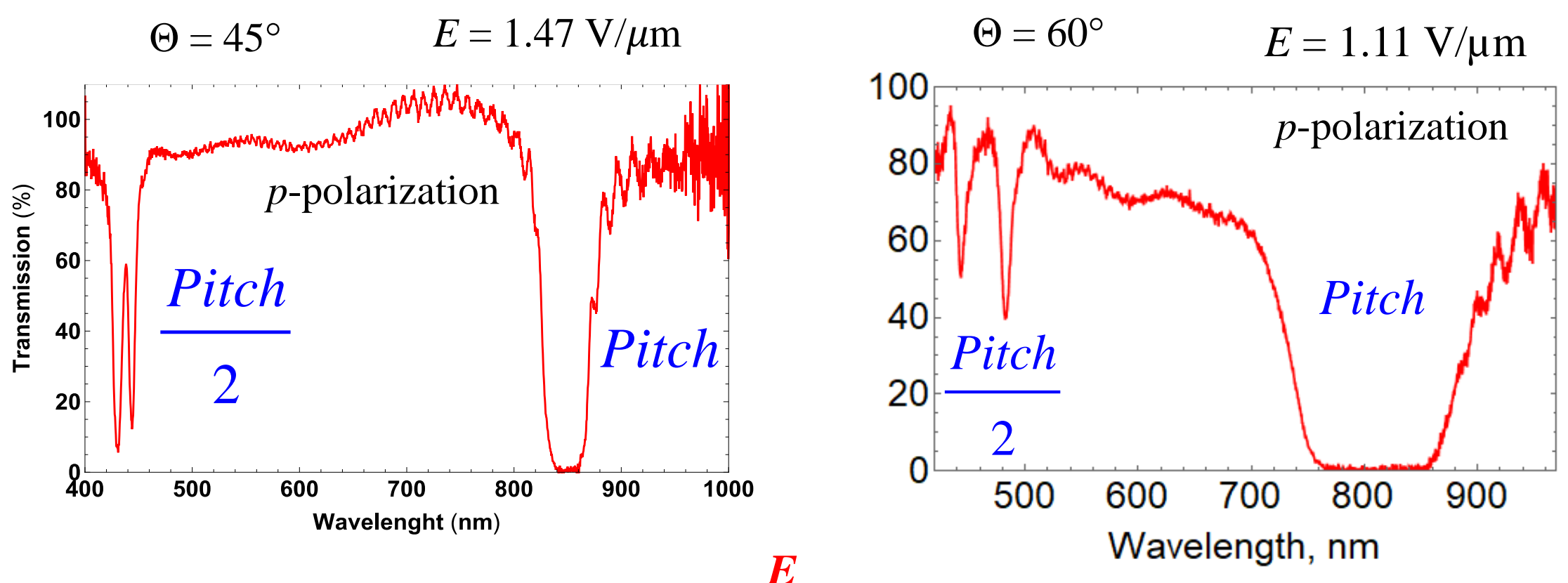

Incidence $\Theta=60^{\circ}$ p-polarization

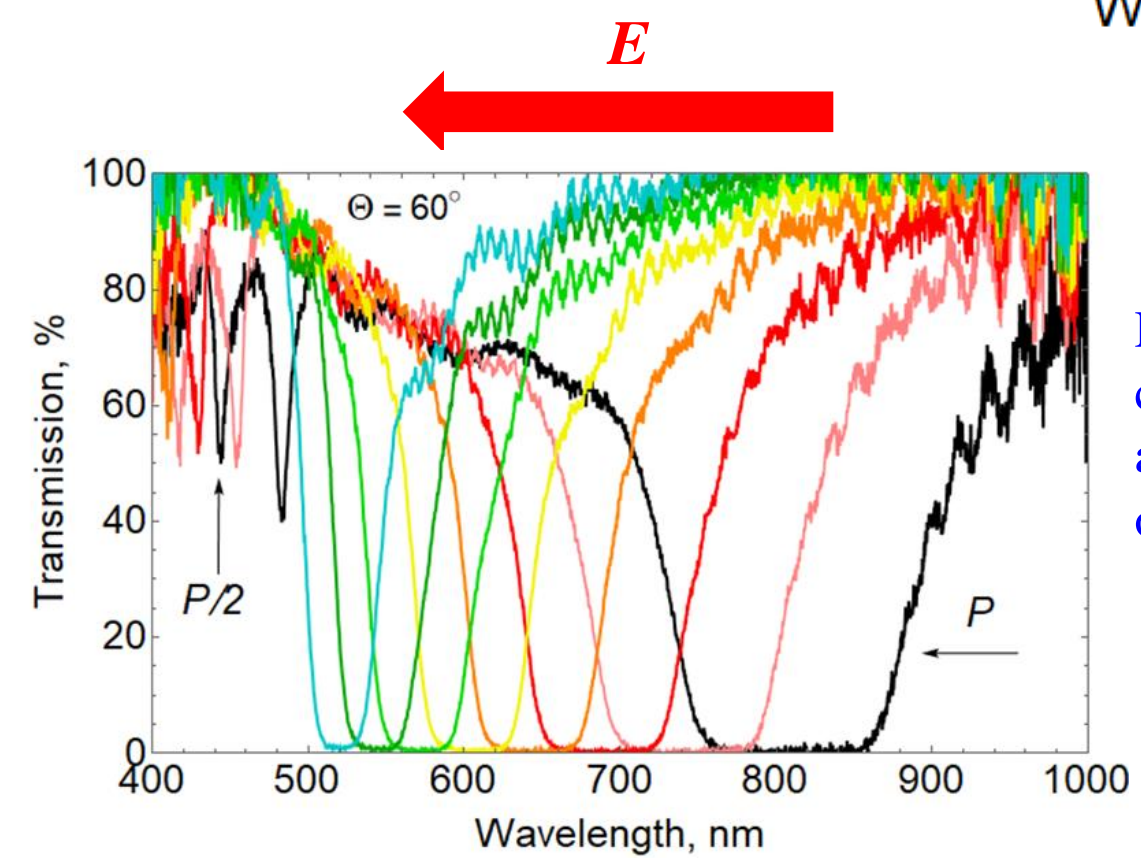

Diffraction at the full pitch $P$ is characterized by a wide bandwidth and total reflection at a large angles of incidence $\Theta$ 


\section{Oblique incidence: polarization characteristics}


The full pitch bandgap is a singlet, polarization independent

The half-pitch is a triplet whose lateral peaks are polarization dependent, while the central peak is not. 


\section{Summary}

- Oblique helicoidal cholesteric $\left(\mathrm{Ch}_{\mathrm{OH}}\right)$ state is continuously tunable by applied field; the single-harmonic periodicity is preserved

- Bragg reflection from $\mathrm{Ch}_{\mathrm{OH}}$ is sensitive to the surface alignment: a blue shift of spectra is observed in homeotropic cells and red shift is observed in planar cells

- Bragg spectra at the $\mathrm{Ch}_{\mathrm{OH}}$ can be used to measure bend elastic constant of the $C h$ phase

- At oblique incidence, diffraction at the half- and full pitch is observed at a varying angle of incidence. Diffraction at the half-pitch is a triplet whose lateral peaks are polarization dependent, while the central peak is not. The full pitch bandgap is a singlet characterized by a wide bandwidth and total reflection at a large angle of oblique incidence 
I want to thank my advisors Dr Oleg Lavrentovich and Dr Sergij Shiyanovskii for all their help and guidance, and my colleagues and friends for fruitful discussions and collaboration, as well as our collaborators for supply of the twist-bend materials.
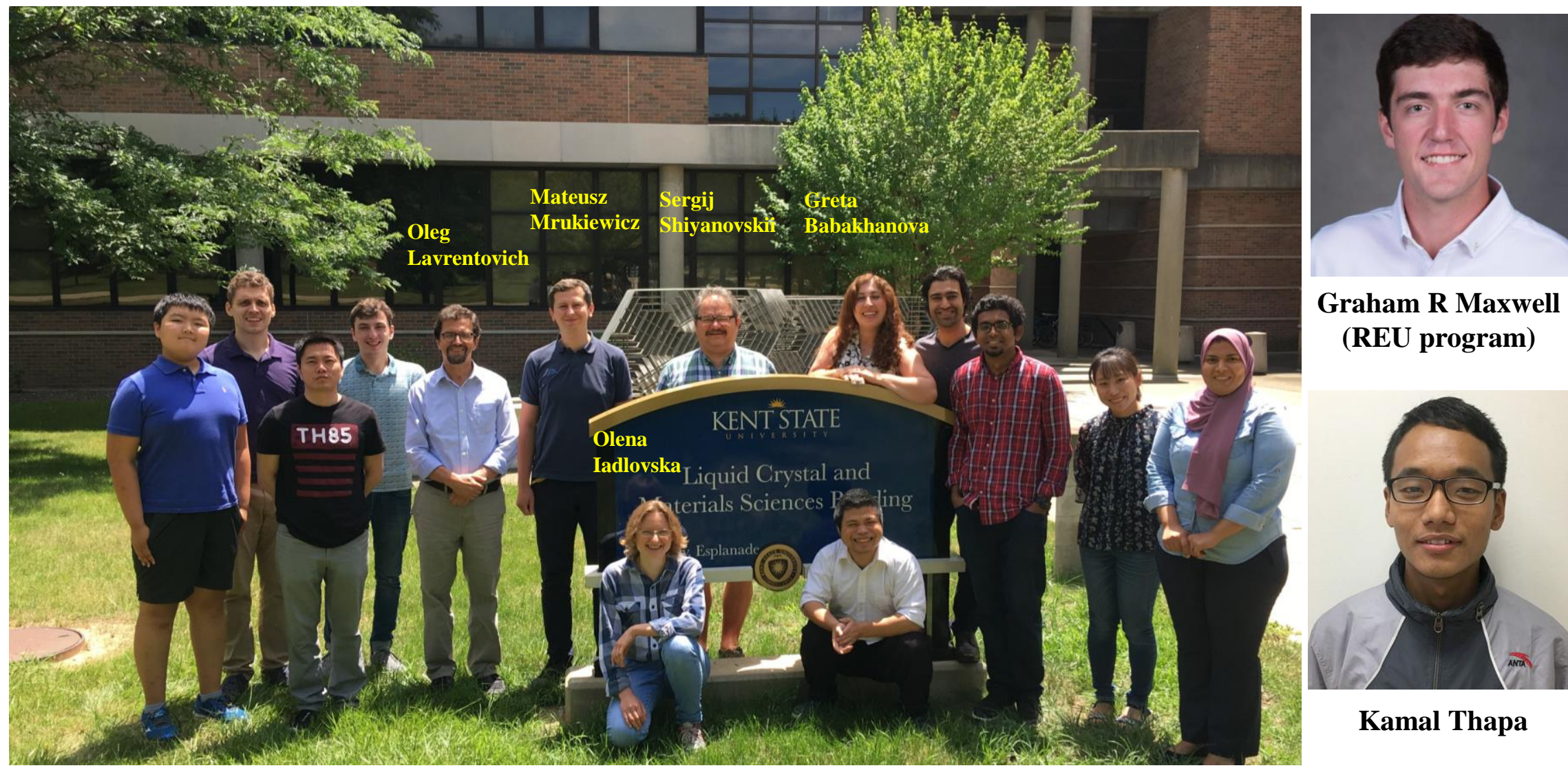

Graham R Maxwell (REU program)

Synthesis of twist-bend materials:

Quan Li and students Hao Wang, Hari K. Bisoyi, Kent State University, USA

Corrie T. Imrie, and students Daniel Paterson, Grant Strachan and Ewan Cruickshank, University of Aberdeen, Scotland, UK Georg H. Mehl, Christopher Welch, University of Hull, UK

National Science Foundation: CHE-1659571 (REU program); ECCS-1906104

MM's visit to Kent State was supported by the Polish Ministry of Science and Higher Education under the Mobilność Plus program (grant no. 1644/MOB/V/2017/0) and the Kosciuszko Foundation exchange program. 